\title{
La educación emocional, su importancia en el proceso de aprendizaje
}

\section{The Emotional Intelligence, its importance in the learning process}

\author{
José Ángel García Retana \\ Universidad de Costa Rica, Sede Guanacaste \\ Guanacaste, Costa Rica \\ jagrucr1058@gmail.com
}

Recibido: 14-XI-2011 • Aceptado: 06-XII-2011 • Corregido: 20-VI-2012

\begin{abstract}
Resumen: Dentro del proceso educativo, se ha tendido a privilegiar los aspectos cognitivos por encima de los emocionales. Incluso para muchos estos dos aspectos deben ser considerados por separado; sin embargo esto no es conveniente si se pretende lograr el desarrollo integral del educando. Para poder incorporar los aspectos emocionales en el proceso educativo se debe caracterizar en qué consisten las emociones, cómo a partir de estas surge la educación emocional y qué papel juega esta dentro de dicho proceso. A su vez, se debe considerar el papel del maestro y cómo las emociones -tanto propias como de los estudiantes-, deben ser tomadas en cuenta en su acto pedagógico; por último se considerará la relación entre emociones $y$ estilos de aprendizaje.
\end{abstract}

Palabras clave: Emociones, educación emocional, cognición, estilos de aprendizaje.

\section{Introducción}

Los procesos de aprendizaje son procesos extremadamente complejos en razón de ser el resultado de múltiples causas que se articulan en un solo producto. Sin embargo, estas causas son fundamentalmente de dos órdenes: cognitivo y emocional. A pesar de esto, el modelo educativo imperante, en general tiende a ignorar o minimizar los aspectos emocionales y en la medida que el educando asciende dentro del mismo, éstos son cada vez menos tomados en cuenta. Sin embargo como resultado de la revolución educativa generada a partir del surgimiento del constructivismo y el impacto de la teoría de las inteligencias múltiples, se ha abierto un nuevo debate en pedagogía que incluye el papel de las emociones como uno de los aspectos 


\begin{abstract}
Within the learning process, cognitive aspects have been privileged over emotional aspects. For some experts, both aspects should be considered separately; however, it might not be suitable if we are to achieve overall development of the learner. In order to accommodate emotional aspects in the educational process, they should be categorized as what they consist on, how they help emotional education to take place and their role as components of the learning process. Additionally, teachers' roles as well as teacher and student emotions should be considered as they affect their own learning processes. Finally, the relationship between emotions and learning styles is to be considered.
\end{abstract}

Keywords: learning process, emotional and cognitive aspects, learning styles fundamentales a ser considerados en la formación integral del educando.

Como es propio en las ciencias sociales, definir constructos teóricos con los cuales trabajar no es tarea fácil, y las emociones no escapan a este dilema. Nadie pone en duda que todos los seres humanos experimentamos su existencia, aunque no siempre podemos controlar sus efectos, de ahí la importancia de considerar a la educación emocional como un aspecto tan importante en la formación del individuo como lo es la educación académica, por constituir ambas un todo tan íntimamente ligada una a la otra, que es impensable considerar la posibilidad de desarrollar cualquiera de estos aspectos por separado.

$\mathrm{Y}$ dado que el acto educativo solo es posible gracias a la presencia de sus dos actores principales, los educandos y los educadores, se debe considerar como los segundos influyen sobre las emociones y los sentimientos de los primeros, dentro de un contexto cultural específico, que es en última instancia el lugar donde se define lo que asumimos como emociones y sentimientos.

\section{Las emociones}

Definir qué son las emociones, es extremadamente difícil y complicado, dado que son fenómenos de origen multicausal. Estas se asocian a reacciones afectivas de aparición repentina, de gran intensidad, de carácter transitorio y acompañadas de cambios somáticos ostensibles, las cuales se presentan siempre como respuesta a una situación de emergencia o ante estímulos de carácter sorpresivo o de gran intensidad, las mismas se vinculan con las necesidades biológicas y bajo el control de las formaciones subcorticales (Bustamante, 1968, como se cita en Martínez, 2009), lo que hace que se presenten de diversas formas y cumplan funciones determinadas generando distintas consecuencias (Puente, 2007). 
La emociones predisponen a los individuos a una respuesta organizada en calidad de valoración primaria (Bisquerra, 2001), esta respuesta puede llegar a ser controlada como producto de una educación emocional, lo que significa poder ejercer control sobre la conducta que se manifiesta, pero no sobre la emoción en sí misma, puesto que las emociones son involuntarias, en tanto las conductas son el producto de las decisiones tomadas por el individuo (Casassus, 2006).

Esto significa que, las emociones son eventos o fenómenos de carácter biológico y cognitivo, que tienen sentido en términos sociales. Se pueden clasificar en positivas cuando van acompañadas de sentimientos placenteros y significan que la situación es beneficiosa, como lo son la felicidad y el amor; negativas cuando van acompañadas de sentimientos desagradables y se percibe la situación como una amenaza, entre las que se encuentran el miedo, la ansiedad, la ira, hostilidad, la tristeza, el asco, o neutras cuando no van acompañadas de ningún sentimiento, entre las que se encuentra la esperanza y la sorpresa (Casassus, 2006). Las emociones se clasifican además según la respuesta que brinda el sujeto como de alta o baja energía, por último, es importante destacar que es posible que se manifiesten distintas emociones a la vez (Santrock, 2002).

A partir de lo anterior es comprensible que existan diferentes opiniones con respecto a lo que son las emociones, incluso algunos/algunas especialistas en el tema utilizan de manera intercambiable los términos emoción y afecto. En el presente ensayo se considera que las emociones se refieren a estados temporales que incluyen el humor y la disposición general (Ormrod, 2005), en calidad de respuestas específicas ante hechos determinados, por lo general de corta duración y relativamente intensa; en tanto los sentimientos y estados de ánimo son de menos intensidad y a diferencia de las emociones pueden durar más tiempo (Guerrit y Zimbardo, 2005).

\section{La inteligencia emocional}

Cuando a finales de los años 80, Howard Gardner publicó Frames of Mind en 1983 y posteriormente Inteligencias Múltiples, La Teoría en la Práctica (1995), planteando la existencia de diferentes inteligencias, incluyendo entre estas las inteligencias intrapersonal e interpersonal, abrió un espacio fundamental en la reconceptualización de la educación, y aunque no era esta su intención, esto llevó a tener que reconsiderar el papel que las emociones juegan en ella. Sin embargo fue Daniel Goleman (1996), quien frente al anquilosado sistema educativo heredado desde la ilustración y determinado por el conductismo, popularizó por medio de su libro, la Inteligencia Emocional (IE), las ideas audaces e innovadoras que Peter Salovey y John Mayer habían propuesto desde 1990, las cuales venían a cubrir los espacios vacios dejados por el constructivismo y el construccionismo como propuestas cognitivistas opuestas al conductismo.

Peter Salovey y John Mayer en 1990 (Dueñas, 2002), plantearon que la IE consistía en la capacidad que posee y desarrolla la persona para supervisar tanto sus sentimientos y emociones, como los de los demás, lo que le permite discriminar y utilizar esta información para orientar su acción y pensamiento. Esta propuesta vino a cuestionar los modelos educativos que hasta finales del siglo $\mathrm{XX}$ insistieron en la construcción de una educación que privilegiaba los aspectos intelectuales y académicos, considerando que los aspectos emocionales y sociales correspondían al plano privado de los individuos (FernándezBerrocal \& Ruiz, 2008). Posteriormente Salovey y Mayer en 1997 (Dueñas, 2002) reformularon su definición anterior para proponer que la IE conlleva la habilidad para percibir con precisión, valorar y expresar emociones, así como el poder acceder y/o generar sentimientos cuando estos facilitan el pensamiento, lo que posibilita conocer comprender y regular las emociones, lo que 
promueve el crecimiento emocional e intelectual (Salovey y Mayer, 1997, citados por Dueñas, 2002), planteándose la posibilidad de su educación.

De esta manera se inició un ardua lucha por combatir el carácter "anti-emocional" del modelo de escuela que imperó hasta antes del siglo XXI, en el cual las emociones fueron formalmente suprimidas con la finalidad de facilitar controlar el tiempo, la mente, el cuerpo y, sobre todo, las emociones de los y las educandos, aduciendo que entre la razón y la emoción existía un universo de distancia (Casassus, 2006) constituyéndolas en opuestos dentro de la existencia humana.

A partir de esto, la escuela anti-emocional definió una diferencia sustantiva entre el pensamiento racional y emocional, tipificando al primero como "objetivo" y asignando al segundo un carácter "subjetivo". Esto se debió a que el pensamiento racional ha sido ligado históricamente con la lógica y particularmente las matemáticas, en virtud de la herencia de la de la cultura griega en el mundo occidental, la cual privilegió aquellas formas de pensar donde la lógica aristotélica se definió como razonamiento correcto. Contrario a esto se ha considerado que el pensamiento emocional y la consecuente conducta emocional, conlleva a una conducta desordenada; sin embargo esto es un craso error, puesto que ha sido demostrado que tal conducta consiste en un sistema organizado, que la misma tiende a una meta, de manera tal que cada emoción conscientemente asumida, es una respuesta articulada con la que cuentan las personas ante determinados estímulos (Sartre, 1987).

El planteamiento de que las emociones están ligadas a factores subjetivos refleja que no se ha tomado en cuenta que éstas incluyen una evaluación de las implicaciones que la situación o evento tiene para el organismo que le permite efectuar una valoración de la situación en la que entran en juego las diferencias individuales, las cuales pueden estar sujetas a un procesamiento controlado o voluntario de la información (Pérez \& Redondo, 2006), que orienta la toma de decisiones partiendo de un chequeo de atribución causa-efecto, discrepancia entre lo ocurrido y lo esperado, la facilitación de metas, la urgencia de responder ante el evento que esta siendo valorado (Jiménez \& Mallo, 1989), interviniendo en todos estos aspectos la memoria, la motivación y el razonamiento, lo que revela que la toma de decisiones esta más afectada por el carácter emocional que racional.

De esta manera, la persona otorga una significación a un evento, por medio de sus emociones, es decir, la emoción es un recurso por el cual, el individuo procura expresar el significado que le otorga a un estímulo aceptado, lo que implica que las emociones remiten a lo que significan, y en su caso incluyen el significado que se le da a la totalidad de las relaciones de la realidad humana, a las relaciones con las demás personas y con el mundo. De ahí que se pueda considerar que una emoción es precisamente una "toma de conciencia del ser humano", que abarca sus diferentes dimensiones bio-psico-sociales, de lo anterior, la conducta racional y la conducta emocional no deben ser vistas como elementos opuestos dado que constituyen dos componentes de la personalidad.

Por lo anterior, cada emoción tiene una finalidad y la percibimos en conductas determinadas y específicas, de manera tal que la misma supone una organización de la conducta, lo cual refleja una toma de conciencia que demanda la existencia de un componente inconsciente para que se pueda, precisamente, tomar consciencia (Casassus, 2006). De esta, las emociones implican una re-significación de los eventos o sucesos, lo que permite comprender y aceptar que la cognición y la emoción se afectan recíprocamente, por lo que la persona que se educa debe ser considerada como una mezcla de razón y emoción, de manera tal que separar estos dos componentes sería atentar contra el carácter humano del ser humano. 
Lo anterior permite reafirmar que la razón y a la emoción son dos aspectos que existen de manera conjunta y por que se hayan inextricablemente unidos en la mente del individuo, lo que los lleva a actuar de manera conjunta, ligados a los conocimientos adquiridos. De hecho, es merced a la necesidad o interés que presenta una persona por adquirir determinados conocimientos, que las emociones y sentimientos influyen en su adquisición, evidenciando que todo lo que hacemos, pensamos, imaginamos o recordamos, es posible por cuanto las partes racional y emocional del cerebro trabajan conjuntamente, mostrando una dependencia una de la otra (Martínez, 2009).

Esta relación, razón-emoción posibilita generar la capacidad adaptativa de la persona, cuya manifestación concreta se aprecia en el poder dar respuestas y soluciones, de manera eficaz, a los problemas que se presentan ligados a las relaciones interpersonales y las conductas disruptivas, propiciando en cambio el bienestar psicológico y el rendimiento académico (Fernández-Berrocal \& Ruiz, 2008).

Es así como a partir de las propuestas de Salovey y Mayer (1990), agregándoles los aportes de Gardner (1995) y Goleman (1996), que es posible considerar la existencia de lo que se puede denominar como Inteligencia Emocional, particularmente al ligar la inteligencia interpersonal con la intrapersonal, las cuales al ser confrontadas con las otras inteligencias que posee el individuo, permiten generar explicaciones plausibles del porqué sujetos de bajo nivel de Coeficiente Intelectual (CI), pero que poseen un alto manejo de sus emociones, son capaces de imponerse ante aquellos sujetos de un nivel de CI más alto, pero de bajo nivel en el manejo de sus emociones (Goleman, 1996).

Esta aparente paradoja se debe a que la IE repercute en todos los ámbitos de la vida del individuo, de ahí la importancia y necesidad de incorporarla en los ámbitos educativos lo que hace necesario un cambio del modelo educativo anti-emocional, demasiado centrado en el conocimiento académico y los contenidos de dominio específico, ya que dicho modelo conlleva un sesgo en el desarrollo de la personalidad del, o la, educando. Surge así la necesidad de un modelo más integral y holístico, que sea capaz de de integrar la educación emocional y la educación académica, como partes inherentes del mismo.

La educación de la afectividad y las emociones, debe ser considerada como una condición primaria para el despliegue de la personalidad (Martínez-Otero, 2007, a), por cuanto constituye parte de un proceso continuo y permanente para lograr el desenvolvimiento de las competencias emocionales como elementos fundamentales, para lograr el desarrollo integral de la persona, posibilitándole al individuo capacitarse para mejorar su calidad de vida, su capacidad de comunicación, aprender a resolver conflictos, tomar decisiones, planificar su vida, elevar su autoestima, incrementar su capacidad de flujo, y sobre todo, desarrollar una actitud positiva ante la vida (Bisquerra, 2005).

En el mundo actual, caracterizado por el dominio de las Tecnologías de la Comunicación y la Información (TIC), donde las posibilidades de comunicación entre las personas han crecido vertiginosamente, afectando irremediablemente todos los órdenes y niveles de la vida de las personas, incluyendo los aspectos cognitivos y emocionales, también han crecido los comportamientos que rayan en lo patológico, como lo son la ansiedad, la depresión, la disciplina, la violencia, los trastornos de la alimentación, etc., los cuales se manifiestan como componentes de un analfabetismo emocional (Dueñas, 2002), y evidencian que tal analfabetismo tiene efectos muy negativos en las personas y la sociedad (Goleman, 1996).

De esta manera, la sociedad en su conjunto y, particularmente, el modelo educativo, deberían tomar consciencia de la importancia de incluir dentro del proceso 
de formación de los individuos a la educación emocional. Tal educación debería implementarse de manera gradual debido a lo complejo de su estructura, y por que demanda un gran esfuerzo de comprensión por parte de los sujetos sobre sí mismos y sobre el entorno (Casassus, 2006), lo que revela que dicha educación está íntimamente ligada a la metacognición. Es así como se puede explicar que las personas que logran una elevada IE, consiguen dominar las manifestaciones de sus emociones, lo que les facilita una mejor adaptación al entorno social y natural (FernándezBerrocal \& Extremera, 2002), lo que permite tener más posibilidades de adaptarse a las diferentes situaciones que se enfrentan y, obtener éxito en los proyectos que se propongan en su vida (Martínez-Otero, 2006).

El modelo educativo que considere la educación emocional como una de sus partes deberá percibir al sujeto desde una perspectiva integral, donde su mente y su cuerpo se articulan para capturar el mundo externo e interpretar el interno. De esta manera, la articulación de conocimientos y emociones, se haría con el fin de procurar que los individuos sean capaces de generar pensamientos que permitan interpretaciones y juicios de valor, como manifestaciones de su consciencia, definiendo sus patrones de conducta (valores), de manera tal que sus emociones se constituyan en los elementos movilizadores que establezcan las acciones a tomar, permitiendo identificar sus intenciones racionales y mantener su voluntad en razón del alcance de sus propósitos (Casassus, 2006), dentro de los marcos de posibilidades que permitan el desempeño y la conducta, que determinan las posiciones que los individuos asumen con respecto a sus vidas, las cuales deben ascender del sometimiento (propio de los infantes) a la libertad (como condición sine qua non del adulto educado).

De esta manera, en la sociedad contemporánea, caracterizada por el pragmatismo y por la ambición a la posesión de bienes y riquezas, más centrada en el tener, que en el ser, los individuos se enfrentan a estereotipos que pretenden definir sus emociones y sentimientos, ligando estos al concepto de "poseer" como bien supremo y final, generando un efecto de encadenamiento a las cosas y apego a estas, de manera tal que la felicidad (y las emociones positivas asociadas a este sentimiento) están en razón de la posesión y no de la satisfacción de sus necesidades personales, sociales, naturales, pero sobre todo espirituales, de manera tal que la afectividad deja de ser el motivo de la vida psíquica (Martínez-Otero, 2007, b) y se sustituye por el concepto de posesión, aun cuando cada vez es más evidente que la riqueza material y la producción y posesión de tales bienes no garantiza ni la satisfacción ni la felicidad (Fernández-Berrocal \& Ruiz, 2008), esto solo es posible superarlo con una adecuada educación emocional.

\section{Cognición, emoción y aprendizaje}

Durante los últimos treinta años, muchos y muchas especialistas en pedagogía, consideraron que la educación consistía en un proceso cognitivo basado en el procesamiento de la información, donde la actividad mental, como expresión del aprendizaje se da gracias a la existencia de conocimientos previos, el nivel, la cantidad y calidad de la acumulación de estos, los cuales articulándose de una manera creativa son generadores de pensamiento productivo (Woolfolk, 2006). A esto se suma el considerar que el aprendizaje es el resultado de la interacción social por medio de esfuerzos cooperativos dirigidos hacia metas compartidas (Pea, 2001 citado por Salomon, 2001). Esta concepción, implicó un considerable avance con respecto a muchas de las consideraciones de los modelos pedagógicos del siglo XX, pero resultó ser insuficiente para poder explicar el porqué de las dificultades del aprendizaje 
en una época caracterizada por la presencia de las TIC, al no tomar en cuenta que las actividades mentales y la interacción social están mediadas por las emociones y los sentimientos que posean y desarrollen los individuos alrededor de tales aspectos.

A partir de Salovey y Mayer (1990), Gardner (1995) y Goleman (1996), la educación no puede reducirse únicamente a lo académico, a la obtención y procesamiento de la información, al desarrollo estrictamente cognitivo, o a las interacciones sociales, como si éstas se dieran en abstracto, sino que debe abarcar todas las dimensiones de la existencia humana (Dueñas, 2002). Nadie duda que el aprendizaje sea un acto deliberado, por lo que no es ni inconsciente ni arbitrario, sino que se da conforme el individuo se desarrolla y se manifiesta como la capacidad para ejecutar una conducta que previamente no se poseía. Sin embargo esto no dice cómo es que el sujeto alcanza tal conducta o capacidad y mucho menos qué lo motiva a su alcance.

Ya en 1960, Bruner consideró que el aprendizaje involucra tres procesos, que considera son casi simultáneos: la adquisición (que implica información nueva o un refinamiento de la información ya existente), la transformación (que implica el manipular el conocimiento para ajustarlo a las nuevas tareas) y la evaluación (para comprobar si la manera en que manipulamos la información es la adecuada). Para lograr esto, el proceso educativo debe tener en cuenta la predisposición del individuo hacia el aprendizaje (Bruner, 1960), lo que de una $u$ otra manera implica el carácter emocional con que se asume el aprendizaje en si mismo. La adquisición, transformación y evaluación, implican una acumulación de experiencias que son interpretadas y "comprendidas", las cuales están inseparablemente unidas a lo que las personas son y sienten (Bisquerra, 2005).

El aprendizaje, por tanto solo es posible en un entorno social, en el que se construyen las estructuras de conocimiento, denominadas "destrezas", las cuales son cada vez más complejas en tanto se maneje cada vez más información. Así, el proceso de aprendizaje desde la Teoría sociocultural incluyó, cuatro puntos esenciales, (Bruner, 1960; Salomon, 2001; Vigotsky, 2005)

- $\quad$ El desarrollo cognitivo, el cual varía de una persona a otra, así como de una cultura a otra.

- La interacción social, la cual posibilita el crecimiento cognitivo de un individuo gracias al aprendizaje guiado dentro de la Zonas de Desarrollo Próximo (Rico, 2009), lo que permite a los aprendices la construcción del conocimiento.

- La socialización, donde se da la construcción de procesos psicológicos individuales como el habla social, lo que permite la comunicación

- $\quad$ El papel de los adultos, quienes transmiten "herramientas" cognitivas en función de facilitar la adaptación intelectual dentro de la cultura que los aprendices deben internalizar.

Una lectura entre líneas, de los puntos anteriores, evidencia que faltó indicar que el aprendizaje constituye un constructo individual y social que se ve afectado por las apreciaciones y valores que, individual y socialmente, se le atribuyen a las emociones en razón de ser estas construidas en términos culturales y contextuales, aspectos que determinan y regulan, cuales emociones son las apropiadas o aceptadas en razón de la interacción entre el sujeto y el ambiente (Bisquerra, 2001), de manera tal que no hay aprendizajes fuera del espacio emocional (Pekrum, 2000, como se cita en Casassus, 2006), al punto que las emociones son determinantes para facilitar $u$ obstaculizar dichos aprendizajes, los cuales a su vez están determinados por los intereses o necesidades del sujeto, en razón de su interacción con el entorno. De esta manera, debe considerarse que el aprendizaje es el producto cultural de dos vertientes que 
interactúan entre sí de manera dinámica, la racional, ligada a la cognición y, la emocional, ligada a los sentimientos, de forma tal que es difícil, sino imposible, separar lo que corresponde a uno u otro dominio.

Estos aspectos, visto en conjunto, generan un marco conceptual que permite explicar que el pensamiento, aunque parezca ser racional, está cargado de aspectos emocionales, de hecho no existe pensamiento puro, ni racional ni emocional (Casassus, 2006), porque los pensamientos dependen de los intereses o necesidades de las personas, y estos aspectos están mediados con el entorno por medio de las emociones. El pensar en como resolver un problema, y lograrlo, produce sensaciones, emociones y sentimientos positivos (como parte de la realización de la persona), en tanto sucede todo lo contrario en el caso de fracasar, por lo que es posible afirmar que la capacidad para atender y entender las emociones, experimentar de manera clara los sentimientos, poder comprender los estados de ánimo, tanto negativos como positivos, son aspectos que influyen de manera decidida sobre la salud mental del individuo, afectan su equilibrio psicológico y, por ende, su rendimiento académico (Fernández-Berrocal y Ruiz, 2008).

\section{El papel del docente en la educación emocional}

Si se considera que el aprendizaje escolar es una actividad social constructiva que realiza el o la estudiante, particularmente junto con sus pares y el maestro o maestra, para lograr conocer y asimilar un objeto de conocimiento, determinado por los contenidos escolares mediante una permanente interacción con los mismos, de manera tal que pueda descubrir sus diferentes características, hasta lograr darles el significado que se les atribuye culturalmente (García, Escalante, Fernández, Escandón, Mustri, \& Puga, 2000), promoviendo con ello un cambio adaptativo (Therer, 1998), es claro que el papel del docente es clave, máxime si a través del aprendizaje se procura el promover habilidades cognitivas y las capacidades emocionales, que le permitan un aprendizaje autónomo y permanente que puedan utilizarlo en situaciones y problemas más generales y significativos y no solo en el ámbito escolar (SEP, 1993; Hernández y Sancho, 1993, Resnick y Klopfer, 1996, citados por García et al, 2000).

Pero esto solo es posible si se toma en cuenta que la intervención del o la docente es una ayuda insustituible en el proceso de construcción de conocimientos por parte del o la educando, de manera tal que sin la ayuda de este es muy probable que los alumnos y las alumnas no alcancen determinados objetivos educativos (García et al., 2000), por cuanto el maestro o maestra no enseña en abstracto, dejando de lado sus propias emociones y sentimientos sino que, ya sea de manera explícita o implícita, transmite los mismos en cada acto pedagógico que desarrolla. Así, ante un mismo evento y en un mismo momento, la interpretación que haga el profesor o profesora, dependerá del estado de consciencia que haya logrado desarrollar (Casassus, 2006), de manera tal que la percepción que este o esta construya del alumno o alumna, estará ligada a las informaciones cognitivas y emocionales que posea del o la educando.

De ahí que el ejemplo de los educadores y educadoras se constituye en un elemento recurrente por parte de los y las educandos, donde en muchos casos se aprecia como un modelo a imitar. Es conocido que los y las estudiantes tienden en muchas ocasiones a realizar y reproducir lo que dicen y hacen sus maestros y maestras, incluyendo los comportamientos producto de sus emociones, ya sea ante el dominio de conocimiento de imparten o enseñan, o ante las actitudes que asumen frente a la vida, particularmente en el caso niños (Martínez-Otero, 2006). 
Por otro lado, para insertarse con posibilidades de éxito dentro del proceso educativo, el o la educando depende de su propia imagen la cual está ligada a sus emociones y sentimientos, la que a su vez responde en muchas ocasiones a la imagen generada por el o la docente (MartínezOtero, 2006). Así, al participar conscientemente del proceso educativo, el o la educando tiene la oportunidad de confirmar o modificar su propia imagen, como resultado de una interacción social la cual incide directamente en el auto concepto que se construye, que puede ser potenciado o disminuido por la institución, la institucionalidad, y los y las docentes participantes en ella, siendo innegable que los sentimientos del o la educando dependen en gran medida del como sus profesores y profesoras lo traten, donde el auto concepto que el profesor o profesoras tengan de sí mismo también actúa sobre la emociones del o la educando, y donde los éxitos o fracasos de ambos tienen un poder de transferencia hacia otros ámbitos más allá del escolar, lo que significa que un potencial comportamiento fuera del entorno escolar se ve influido por lo vivido en este (Martínez-Otero, 2007, b).

La capacidad de identificar, comprender y regular las emociones es fundamental por parte de los y las profesores, debido a que tales habilidades influyen en los procesos de aprendizaje, en la salud física, mental y emocional de los y las educandos y son determinantes para establecer relaciones interpersonales positivas y constructivas con estos, posibilitando una elevación en su rendimiento académico (Cabello, Ruiz \& Fernández, 2010), ya que, cuando el profesorado inspira confianza y seguridad, e instruye con dominio y confianza, es posible que los y las estudiantes asuman una actitud más empática hacia el o la docente y hacia la disciplina que este o esta imparte (Casassus, 2006). El propio convencimiento del profesorado en lo que hace, es un poderoso recurso para ganar la actitud de los y las educandos, de manera tal que, si el maestro o maestra persigue realmente incidir de manera positiva en sus educandos debe procurar ganar de manera simultánea tanto la "razón" como el "corazón" de estos, de lo contrario sus esfuerzos estarán propensos al fracaso.

\section{Emociones y estilos de aprendizaje}

Para Therer (1998), cuando se conoce como aprenden los y las estudiantes es que el esfuerzo de la enseñanza podría tener algún efecto positivo, este aprender no depende únicamente de las capacidades cognitivas de los y las educandos, sino de sus disposiciones emocionales, dado que el o la docente es más que un mero transmisor de información, es un creador o creadora de espacios de aprendizaje y le corresponde gestionar las condiciones que posibiliten organizar las situaciones de aprendizaje las cuales dependen de al menos cuatro factores ligados a los y las estudiantes: a) su motivación (donde se insertan los aspectos emocionales), b) sus capacidades cognitivas, c) sus estilos de aprendizaje, y d) los objetivos curriculares a ser alcanzados.

Esto lleva a considerar la importancia de poder articular en el proceso de aprendizaje, las emociones de los y las educandos con sus estilos de aprendizaje, entendido este como la preferencia mostrada por el y la educando para abordar un aprendizaje (Popescu, 2008), lo que corresponde a la manera dominante de aprender que manifiestan estos o estas (De Lièvre, Temperman, Cambier, Decamps \& Depover, 2009), reflejando así las disposiciones personales que influyen o influencian en la habilidad para accesar a la información; a lo que habría que adicionar los estilos de enseñanza del profesor o profesora.

$\mathrm{Si}$ el o la docente logran hacer compatibles sus estilos de enseñanza y el estilo de aprendizaje de los y las estudiantes tomando en cuenta las emociones de ambos, probablemente el rendimiento académico 
de estos y estas será mayor, aspecto que redundará en la generación de más emociones positivas, que a su vez potencian un mayor aprendizaje (Felder, 1990 y Pérez, 1995, como se cita en Amado Brito \& Pérez, 2007), construyéndose un círculo virtuoso entre aprendizaje y emociones, de manera tal que el rendimiento académico vendrá por sí solo (Casassus, 2006). De lo contrario, no lograr superar las diferencias entre los estilos de enseñanza del profesorado y los estilos de aprendizaje de los estudiantes, puede constituirse en fuente de conflicto, tensión y malos entendidos (Grasha, 2002).

De esta manera, el estilo de aprendizaje del profesor o profesora repercute en su manera de enseñar, ya que frecuentemente tienden a enseñar como a ellos les gustaría aprender (Gallego y Nevot, 2008), lo cual revela las emociones y sentimientos de los mismos, por lo que deben ser ellos quienes deben informarse sobre los estilos de aprendizaje de sus estudiantes y los mecanismos que posibiliten una educación emocional, en razón de potenciar el círculo virtuoso apuntado anteriormente ya que si los y las docentes ignoran los estilos de aprendizaje de los y las estudiantes, así como las emociones y los sentimientos de estos y estas, el resultado es tan perjudicial como el no dominar la disciplina que se enseña, o no contar con las técnicas y estrategias didácticas que motiven a los y las estudiantes (Bonilla, 1998), generándose entonces apatía, desinterés, reducción de la efectividad del planeamiento didáctico y de las estrategias metodológicas.

El conocer los estados emocionales de los y las estudiantes, así como sus estilos de aprendizaje, puede ayudar al profesor o profesora a organizar de manera más eficaz y eficiente el proceso de aprendizaje-enseñanza a implementar (Thompson \& Aveleyra, 2004), y posibilita atender a los y las estudiantes de manera más personal, guiándolos en el contexto del aprendizaje; solo así es que el profesor o profesora realmente puede contribuir a que sus estudiantes se conviertan en los constructores de sus propios aprendizajes (Thomson \& Mazcasine, 2000).

Lo anterior permite considerar que los niveles de éxito y/o fracaso por parte de los y las estudiantes, en el aprendizaje de cualquier disciplina, podrían estar asociadas, entre otros aspectos a la concordancia/discrepancia entre los estilos de aprender/enseñar, que se dan entre los y las estudiantes y los y las docentes, así como en la comprensión de las emociones y los sentimientos de ambos y cómo éstos afectan directamente al proceso cognitivo; y no exclusivamente a las usuales debilidades que se apuntan en direcciones únicas, como lo son entre otras, estudiantes con bajos niveles de conocimiento, ausencia de conocimientos previos significativos, o bien profesores o profesoras incapaces de lograr una comunicación efectiva, por lo que se puede afirmar que, un educador emocionalmente inteligente y un clima favorable en el aula son factores esenciales para el aprendizaje (Campos, 2010).

\section{Conclusiones}

El paradigma de la educación racional, centrada en el aprendizaje de contenidos, ignorando las dimensiones no académicas de los y las estudiantes, particularmente las emociones, ha mostrado su agotamiento. Por ello se requiere urgentemente un cambio en ese sentido, lo cual solo será posible en la medida que la sociedad revalore el papel de las emociones como un elemento primario, fundamental y sustantivo del proceso de aprendizaje.

Debemos tener claro que no se aprende lo que no se quiere aprender, no se aprende aquello que no motiva, y si algo no motiva se debe a que no genera emociones positivas que impulsen a la acción en esa dirección. Esta es la clave de considerar a las emociones como parte del aprendizaje, por lo cual la educación emocional se constituye, por 
derecho propio, en una necesidad que va más allá del ámbito escolar.

La educación emocional debe ser vista, conceptualizada y puesta en marcha para procurar que los y las educandos se conozcan a sí mismos y conozcan a los demás, se respeten, respeten a los otros y al entorno donde viven, de manera que se pueda plantear el desarrollo integral de su personalidad como requisito para la construcción de la felicidad.

Si bien, las emociones surgen de manera espontánea, por medio de la educación emocional se puede procurar que las mismas no se desboquen y lleven a los y las educandos a cometer actos y mantener conductas que pongan en peligro sus vidas o las de los demás, ya sea en el plano físico o psicológico. El esfuerzo de la educación emocional debe estar orientado a que los y las educandos aprendan a aceptar sus emociones y sentimientos, y a partir de ello, aprendan a decidir que conducta es la más apropiada a ser manejada según las circunstancias, de manera tal que las mismas contribuyan a una interacción social y personal constructiva, positiva, capaz de contribuir a la elevación de su calidad de vida.

El profesorado no constituye un elemento neutro en su acto pedagógico tanto en razón de la transmisión de contenidos de dominio culturalmente especificados (currículo), como por la manifestación de sus emociones y del impacto de éstas en los educandos. De esta manera, el proceso de aprendizaje-enseñanza se ve influido por la forma en que el profesor o profesora logra manejar sus propias emociones y sentimientos con respecto a sí mismo, su disciplina, su concepción del acto educativo, pero sobre todo por la percepción desarrollada por los y las estudiantes a su cargo, de manera tal que las actitudes que el docente asuma pueden contribuir o dificultar el aprendizaje por parte de los educandos.

Por último, dado que la educación debe ser un proceso integral, donde cognición y emoción constituyen un todo, estos dos componentes del proceso educativo no deben ser vistos como los extremos de un intervalo que define la vida de las personas, sus conductas o comportamientos. La cognición y la emoción constituyen un todo dialéctico, de manera tal que la modificación de uno irremediablemente influye en el otro y en el todo del que forman parte. Por ello en el aula muchas veces el aprender depende más de la emoción que de la razón con que se trabajan los objetivos del aprendizaje, y se actúa sobre los mismos. Todo esto nos conduce a señalar que si se gana el corazón del alumno o de la alumna, el aprendizaje está prácticamente asegurado.

\section{Referencias}

Amado, M., Brito, R., Pérez, C. (2007). Estilos de aprendizaje de estudiantes de Educación Superior. Recuperado de www.alammi.info/revista/numero2/pon_0011.pdf

Bisquerra, R. (2001). Educación emocional $y$ bienestar. ( $1^{\mathrm{a}}$ Reimpresión).(2da. ed.) Barcelona, España: Editorial CISS Praxis.

Bisquerra, R. (2005). La educación emocional en la formación del profesorado. Revista Interuniversitaria de Formación del Profesorado, 19 (3). Recuperado de http://redalyc.uaemex.mx/pdf/274/27411927006.pdf

Bonilla, F. (1998). Estilos de Aprendizaje de los estudiantes de la Universidad de Costa Rica. Revista Educación, 12 (1). Universidad de Costa Rica.

Bruner, J. (1960). El proceso de la educación. México: Unión Tipográfica Editorial Hispano-Americana.

Cabello, R., Ruiz, D., Fernández, P. (2010). Docentes emocionalmente inteligentes. Revista Electrónica Interuniversitaria de Formación del Profesorado. 13 (1). Recuperado de http://dialnet.unirioja.es/servlet/ articulo? codigo $=3163455$ 
Campos, A. (2010). Neuroeducación: uniendo las neurociencias y la educación en la búsqueda del desarrollo humano. La Educación. Revista Digital, 143. Recuperado de www.educoea. org/...Educacion.../laeducacion.../neuroeducacion.pdf

Casassus, J. (2006). La educación del ser emocional. (1a ed.). Universidad Virtual del Instituto Tecnológico de Monterrey, México: Ediciones Castillo,

De Lièvre, B., Temperman G., Cambier, J., Decamps, S., Depover, Ch. (2009). Analyse de l'influence des styles d'apprentissage sur les interactions dans les forums collaboratifs. Recuperado de http://w3.u-grenoble3. fr/.../epal2009-delievre-et-al.pdf

Dueñas, M. (2002). Importancia de la inteligencia emocional: un nuevo reto para la orientación educativa. Educación, XXI, (005) Recuperado de http://redalyc.uaemex.mx/src/inicio/ ArtPdfRed.jsp?iCve=70600505

Fernández-Berrocal, P. y Ruiz, D. (2008). La inteligencia emocional en la Educación. Education \& Psychology. Recuperado de http://orientacion. educa.aragon.es/admin/admin_1/ file/.../A_contador.pdf

Fernández-Berrocal, P. y Extremera, N. (2002). La inteligencia emocional como una habilidad esencial en la escuela. Revista Iberoamericana en Educación. Recuperado de http://www.rieoei.org/ deloslectores/326Berrocal.pdf

Jiménez, A. y Mallo, M. (1989). Reconocimiento de emociones a partir de descripciones verbales. Revista de Psicología Social, 4 (1). Recuperado de http://dialnet.unirioja.es/servlet/ articulo? codigo $=2903501$

García, L., Escalante, L., Fernández, L.G., Escandón, M.C., Mustri, A. y Puga, I. (2000). Proceso de EnseñanzaAprendizaje. Documento de trabajo SEP. Recuperado de http://www. white.oit.org.pe/spanish/260ameri/ oitreg/activid/.../actrav/.../archivo47. pdf

Gallego, D. y Nevot, A. (2008). Los estilos de aprendizaje y la enseñanza de las matemáticas. Revista Complutense de Educación, 19 (1). Recuperado de http://dialnet.unirioja.es/servlet/ revista?codigo $=1127$

Gardner, H. (1995). Inteligencias Múltiples. La teoría en la práctica. Barcelona. España: Ediciones Paidós Ibérica, S. A.

Grasha, A. (2002). Teaching with style. A practical guide to enhancing learning by understanding teaching and learning Styles. Recuperado de http://www.ilte.ius.edu/pdf/teaching_with_style.pdf

Guerrit R. y Zimbardo, P. (2005). Psicología $y$ vida. (17). México: Pearson Educación.

Goleman, D. (1996). La inteligencia emocional. Javier Vergara (Ed.). Santa Fe de Bogotá, Colombia.

Martínez, C. (2009). Consideraciones sobre inteligencia emocional. La Habana, Cuba: Editorial Científico-Técnica.

Martínez-Otero, V. (2006). Fundamentos e implicaciones educativas de la inteligencia afectiva. Revista Iberoamericana de Educación, 39 (2). Recuperado de http://www.rieoei.org/ deloslectores/1349Martinez.pdf

Martínez-Otero, V. (2007, a). Psicología de la inteligencia afectiva: implicaciones pedagógicas. Revista Electrónica de Psicología. Recuperado de http:// dgsa.uaeh.edu.mx/revista/psicologia/ IMG/pdf/No.4-15.pdf

Martínez-Otero, V. (2007, b). La inteligencia afectiva. ( ${ }^{\mathrm{a}}$. ed.). Universidad Virtual del Instituto Tecnológico de Monterrey, México: Editorial CCS.

Ormrod, J. (2005). Aprendizaje humano. (4 ${ }^{\mathrm{a}}$. ed.). España. Pearson Educación

Pérez, M. y Redondo, M. (2006). Procesos de valoración y emoción: características, desarrollo, clasificación y estado actual. Revista Electrónica 
de Motivación y Emoción, 9 (22). Recuperado de http://www.infocop. es/view_article.asp?id=1181

Popescu, E. (2008). Dynamic adaptive hypermedia systems for e-learning. Doctorat Tis. Université de Craiova, Roumanie.Recuperado desde http://www.tel.archives-ouvertes.fr/docs/00/34/34/60/PDF/thesis_ ElviraPopescu.pdf

Puente, A. (2007). Cognición y Aprendizaje. Fundamentos Psicológicos. España: Editorial Pirámide.

Rico, P. (2009). La Zona de Desarrollo Próximo. Procedimientos y tareas de aprendizaje. La Habana, Cuba: Editorial Pueblo y Educación.

Salomon, G. (Comp.). (2001). Cogniciones distribuidas. Consideraciones psicológicas y educativas. Buenos Aires, Argentina: Amorrortu Editores.

Santrock. J. (2002). Psicología de la Educación. México : McGraw-Hill.

Salovey, P. y Mayer, J. (1990) Emotional Inteligence. Baywood Publishing Co. Inc. Recuperado de http:// w w w.unh.edu/.../EI $1990 \% 20$ Emotional\%20Intel...
Sartre, Jean P. (1987). Bosquejo de una teoría de las emociones. (5 ${ }^{a}$ reimp.) Madrid, España: Alianza Editorial.

Therer, J. (1998). Styles d'enseignement, styles d'apprentissage et pédagogie différenciée en sciencies. Informations Pédagogiques, 40. Recuperado de http://www.restode.cfwb.be/download/ infoped/info40a.pdf

Thompson, S. y Aveleyra, E. (2004.). Estilos de aprendizaje en matemáticas. Recuperado de http://www.fceco. uner.edu.ar/cpn/catedras/matem $1 /$ educmat/em22ta.doc.

Thompson, B. y Mazcasine, J. (2000). Attending to Learning Styles in Mathematics and Science Classrooms. ERIC Digest. Recuperado de http:// www.ericdigests.org/2000-1/attending.html

Vigotsky, L. (2005). Pensamiento y lenguaje. La Habana, Cuba : Editorial Pueblo y Educación

Woolfolk. A, (2006). Psicología Educativa. ( $9^{\mathrm{a}}$ ed.). México : Pearson Educación. 